\title{
Subarachnoid and Intracerebral Hemorrhage Associated with Necrotizing Angiitis Due to Methamphetamine Abuse
}

\author{
-An Autopsy Case-
}

\author{
Shobu SHIBATA, Kazuo MORI, Ichiro SeKINE* and Hirofumi SUYAMA** \\ Departments of Neurosurgery and **Legal Medicine, and *Department of Pathology, \\ Atomic Disease Institute, Nagasaki University School of Medicine, Nagasaki
}

\begin{abstract}
The authors report an autopsy case of methamphetamine-related intracranial hemorrhage and vasculitis. A 22-year-old female was comatose after an intravenous injection of an unknown dose of methamphetamine. Computed tomographic scans demonstrated massive subarachnoid hemorrhage and hematoma in the corpus callosum. Cerebral angiography revealed nonfilling of bilateral intracranial carotid arteries and extravasation of contrast medium from the right pericallosal artery which was visualized retrogradely via the vertebral artery. Postmortem studies found cerebral edema, subarachnoid, intraventricular, and intracerebral hemorrhage, and intracranial vasculitis, but no aneurysm or arteriovenous malformation. Necrosis of vessel walls with destruction of the smooth muscle layer, but no leukocytotic infiltration of the vessel walls were observed in all major cerebral arteries. The hemorrhage probably resulted from medial necrosis in the large intracerebral vessels, and a sudden drug-induced rise in blood pressure.
\end{abstract}

Key words: cerebral angiitis, hemorrhage, methamphetamine

\section{Introduction}

Increasing methamphetamine abuse worldwide has aroused widespread concern in medical and governmental agencies. Cerebral angiitis and intracranial hemorrhage are reported to associate with methamphetamine abuse. ${ }^{1-3,5-7,10,12,13,16-19)}$ The two major factors involved are the drug hypertensive effect and a pre-existing vascular lesion. Here we report an autopsy case of methamphetamine-related intracranial hemorrhage and vasculitis. The relationship between drug abuse and occurrence of intracranial bleeding or cerebral vasculitis is discussed.

\section{Case Report}

A 22-year-old female suffered a general seizure with complete loss of consciousness immediately after intravenous injection of an unknown dose of methamphetamine. She had a history of intravenous metham-

Received

April 9, 1990;

Accepted June 7, 1990 phetamine abuse of undeterminate duration until 1 year previously.

On admission, she was deeply comatose. Her vital signs were blood pressure $160 / 100 \mathrm{mmHg}$, pulse rate $81 / \mathrm{min}$, respiration rate $24 / \mathrm{min}$. Laboratory studies showed normal blood count, biochemistry, platelet count, and coagulation levels. The blood methamphetamine level was $37.4 \mu \mathrm{g} / 100 \mathrm{gm}$.

Computed tomographic (CT) scans demonstrated massive subarachnoid hemorrhage and hematoma in the corpus callosum (Fig. 1). Cerebral angiography revealed nonfilling of the bilateral intracranial carotid arteries, and extravasation of contrast medium from the right pericallosal artery which was visualized retrogradely via the vertebral artery (Fig. 2). Respiratory arrest occurred 13 hours after onset, so she was intubated and ventilated. She died 51 hours after onset.

An autopsy discovered subarachnoid and intraventricular hemorrhage and a callosal hematoma, originating from a rupture in the lower surface of the right pericallosal artery. Uncal and transtentorial her- 


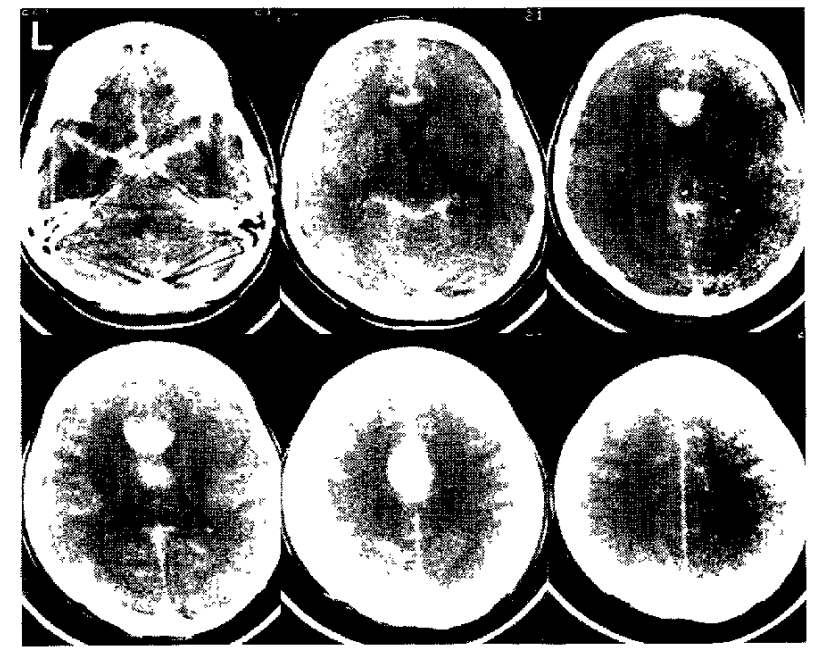

Fig. 1 CT scans showing massive subarachnoid hemorrhage and hematoma in the corpus callosum.

niation were evident. Examination of the cerebral vessels found no evidence of aneurysm or arteriovenous malformation. All cerebral arteries and their major branches possible were serially transected. Figure 3 shows the location and number of sections

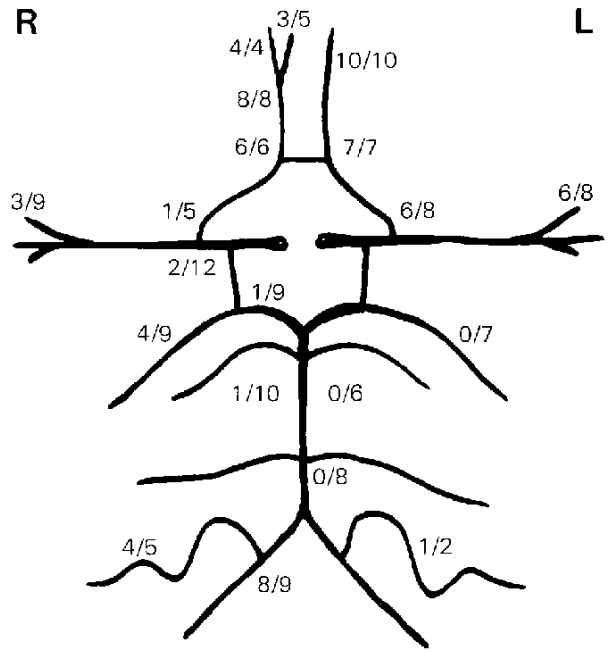

Fig. 3 Distribution of medial necrosis. Numerals indicate the number of sections examined (denominator) and the number of sections demonstrating necrotic media (numerator) in 20 segments.

examined. Histologically, many artery cross-sections showed various extents of medial necrosis. Necrotic lesions demonstrated absence of nuclei, atrophy
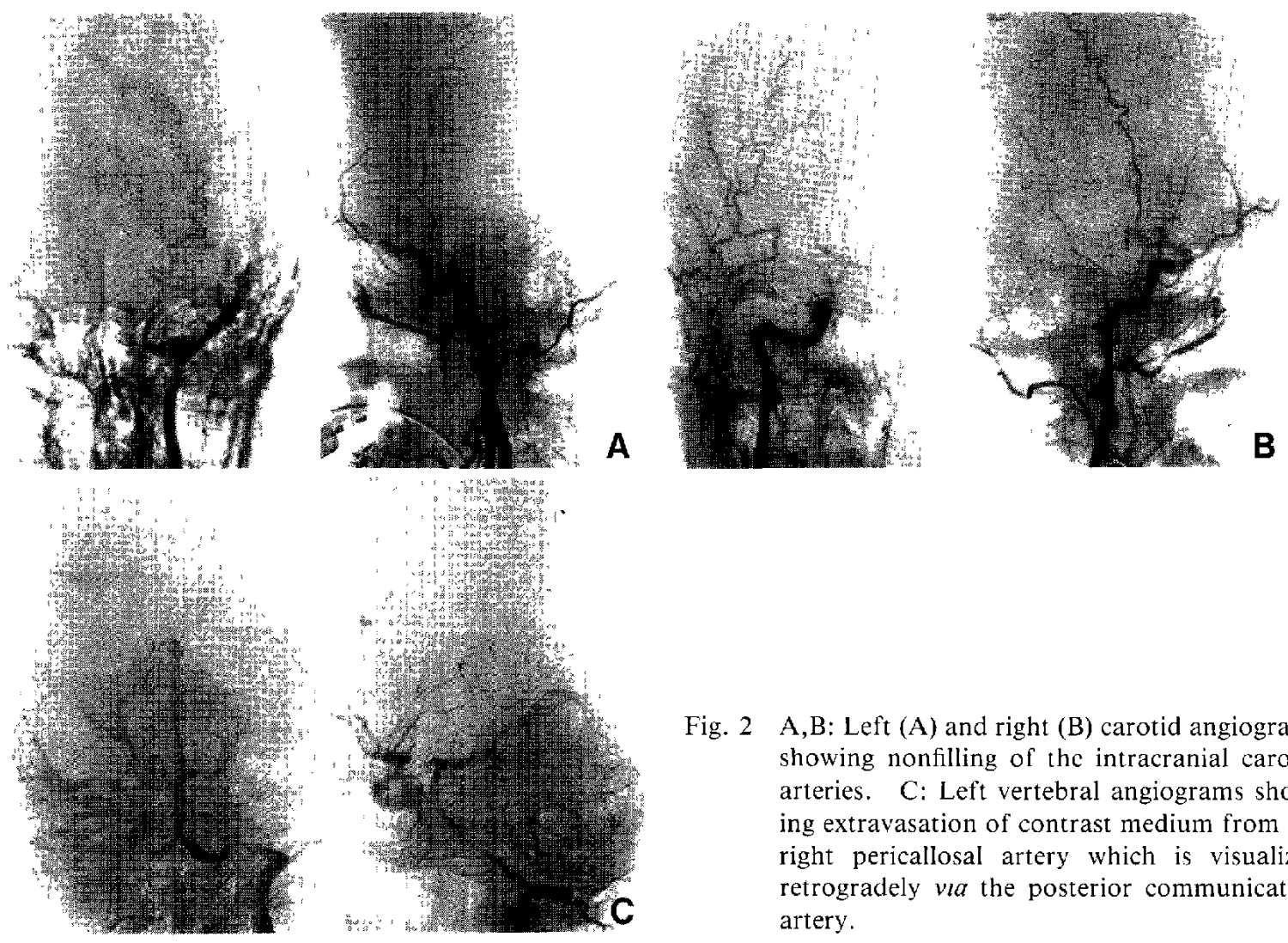

Fig. 2 A,B: Left (A) and right (B) carotid angiograms showing nonfilling of the intracranial carotid arteries. C: Left vertebral angiograms showing extravasation of contrast medium from the right pericallosal artery which is visualized retrogradely via the posterior communicating artery. 

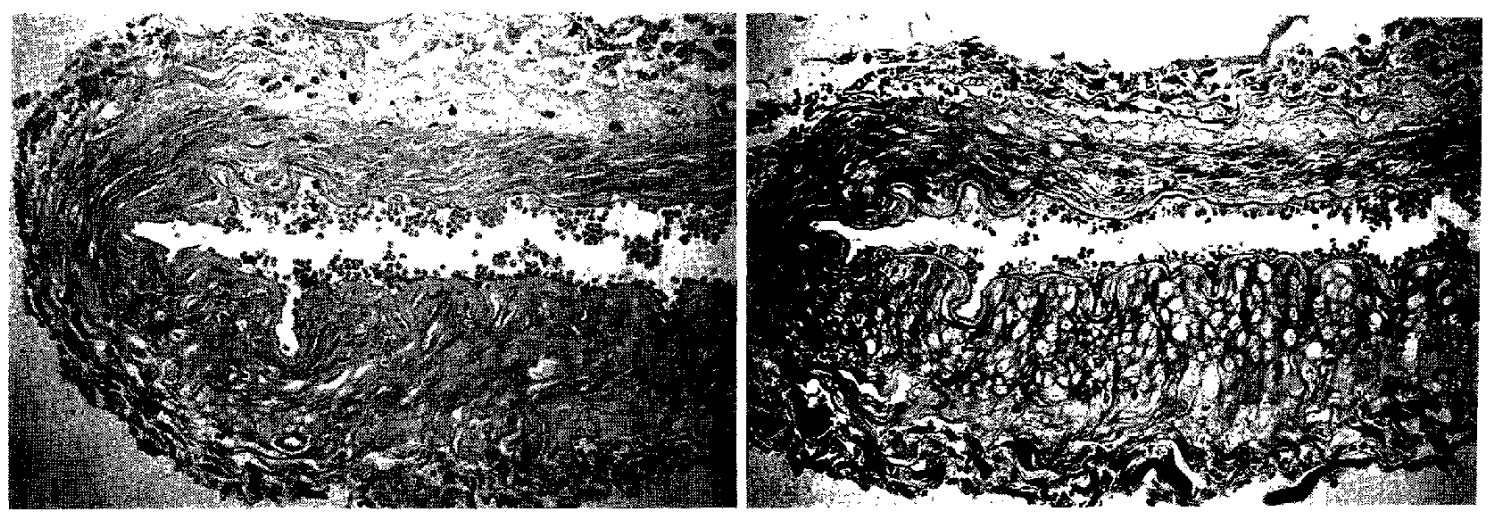

Fig. 4 Photomicrographs of anterior cerebral artery showing thinning and necrosis of medial smooth muscle cells in the upper half. HE (left) and Mallory-azan (right) stains, $\times 250$.

with increased eosinophilic smooth muscle character, and decreased medial thickness. No inflammatory cells indicating degenerating polymorphonuclear leukocytes were present. No defect in the intima and internal elastica layers could be demonstrated with special stains (Fig. 4). Necrosis of the smooth muscle layer was greatest in the anterior cerebral artery, and gradually decreased in severity in the middle cerebral, vertebral, posterior cerebral, and was least in the basilar arteries. Such changes were not observed in the small branches.

\section{Discussion}

Citron et al ${ }^{4,8)}$ reported 14 cases of necrotizing angiitis associated with drug abuse. The drugs included narcotics, hallucinogens, stimulants, and sedatives. Methamphetamine was the most common. The vascular necrotizing angiitis observed was characterized by microaneurysms, indistinct vessel outlines, segmental luminal irregularities, and thrombosis in the lung, kidney, liver, pancreas, and small bowel vessels. These changes occurred mainly in the medium and small arteries in most body organs and the arterioles of the brain. Fibrinoid necrosis was apparent in the media and intima, with cellular infiltration. Such changes are indistinguishable from periarteritis nodosa, but differed from hypersensitivity angiitis, which frequently involves small arteries, capillaries, and venules.")

Margolis and Newton ${ }^{11}$ described pronounced irregularity and beading in the middle and anterior cerebral arterial branches after intravenous methamphetamine administration. Rumbaugh et al..$^{14,151}$ reported "beading" on cerebral arteriograms in 19 drug-abuse patients presenting with seizures, hemiparesis, or stupor, but excluding patients with intracranial hemorrhage. Histological studies of two patients indicated vasculitis. "Beading" is a rather nonspecific arteriographic finding with various causes. In the rhesus monkey, arteriography demonstrated "beading" almost immediately after intravenous methamphetamine administration. The changes were reversible as clearly demonstrated by serial selective carotid angiography after a month of prednisone treatment and drug abstention. This clinical and experimental evidence suggests methamphetamine has a direct toxic or immunological effect on the cerebral vasculature.

In our patient, autopsy confirmed massive subarachnoid hemorrhage and callosal hematoma due to rupture of the right pericallosal artery. Histological examination found necrosis of the vessel walls with destruction of the smooth muscle layer, but no leukocytic infiltration of the vessel wall. These are characteristic changes of acute necrotizing vasculitis, and were observed in all major arteries; anterior cerebral, middle cerebral, vertebral, posterior cerebral, and basilar arteries; but not the small branches. Detailed gross and microscopic examination of the cerebral vessels revealed no aneurysms or arteriovenous malformations.

\section{References}

1) Bessen HA: Intracranial hemorrhage associated with phencyclidine abuse. JAMA 248: 585-586, 1982

2) Cahill DW, Knipp H, Mosser J: Intracranial hemorrhage with amphetamine abuse. Neurology (NY) 31: 1058-1059, 1981

3) Chynn KY: Acute subarachnoid hemorrhage. JAMA 233: 55-56, 1975

4) Citron BP, Halpern M, MacCarron M, Lundbery GD, McCormick R, Pingus IJ, Tatter D, Haverback BJ: Necrotizing angiitis associated with drug abuse. 
N Engl J Med 283: 1003-1011, 1970

5) Delaney $\mathbf{P}$, Estes $M$ : Intracranial hemorrhage with amphetamine abuse. Neurology (NY) 30: 1125-1128, 1980

6) Edwards KR: Hemorrhagic complications of cerebral arteritis. Arch Neurol 34: 549-552, 1977

7) Goodman SJ, Becker DP: Intracranial hemorrhage associated with amphetamine abuse. JAMA 212: 480-481, 1970

8) Halpern M, Citron BP: Necrotizing angiitis associated with drug abuse. $A J R$ 111:663-671, 1971

9) Kessler JT, Jortner BS, Adapon BD: Cerebral vasculitis in a drug abuser. J Clin Psychiatry 39: 559564, 1978

10) Luke SA: Intracerebral hemorrhage from an arteriovenous malformation after amphetamine injection. Arch Neurol 40: 60-61, 1983

11) Margolis MT, Newton TH: Methamphetamine (speed) arteritis. Neuroradiology 2: 179-182, 1971

12) Ogasawara K, Ogawa A, Kita H, Kayama T, Sakurai Y, Suzuki J: Intracerebral hemorrhage and characteristic angiographic changes associated with methamphetamine: A case. No To Shinkei 38: 967-971, 1986 (in Japanese)

13) Olsen ER: Intracranial hemorrhage and amphetamine usage. Angiology 28: 464-471, 1977

14) Rumbaugh CL, Bergeron RT, Fang $\mathrm{HCH}$,
McCormick R: Cerebral angiographic changes in the drug abuse patient. Radiology 101: 335-344, 1971

15) Rumbaugh CL, Bergeron RT, Scanlan RL, Teal JS, Segall HD, Fang HCH, McCormick R: Cerebral vascular changes secondary to amphetamine abuse in the experimental animal. Radiology 101: 345-351, 1971

16) Salanova $V$, Taubner R: Intracerebral haemorrhage and vasculitis secondary to amphetamine use. Postgrad Med J 60: 429-430, 1984

17) Weiss SR, Raslind R, Morganstern NL, Pytlyk PJ, Baiz TC: Intracerebral and subarachnoid hemorrhage following use of methamphetamine (speed). Int Surg 53: 123-127, 1970

18) Yarnell PR: Speed: Headache and hematoma. Headache 17: 69-70, 1977

19) Yu YJ, Cooper PR, Wellenstein DE, Block B: Cerebral angiitis and intracerebral hemorrhage associated with methamphetamine abuse. $J$ Neurosurg 58 : 109-111, 1983

Address reprint requests to: S. Shibata, M.D., Department of Neurosurgery, Nagasaki University School of Medicine, 7-1 Sakamoto-machi, Nagasaki 852, Japan. 